Schweizerisches Jahrbuch für Entwicklungspolitik

$22-2 \mid 2003$

Informationsgesellschaft und internationale

Zusammenarbeit: development.com

\title{
Aussichten auf virtuellen Wohlstand
}

Marie Thorndahl

\section{OpenEdition}

1 Journals

Electronic version

URL: http://journals.openedition.org/sjep/518

DOI: $10.4000 /$ sjep.518

ISSN: 1663-9677

Publisher

Institut de hautes études internationales et du développement

\section{Printed version}

Date of publication: 1 novembre 2003

Number of pages: 3-27

ISSN: 1660-5926

\section{Electronic reference}

Marie Thorndahl, « Aussichten auf virtuellen Wohlstand », Schweizerisches Jahrbuch für

Entwicklungspolitik [Online], 22-2 | 2003, Online erschienen am: 10 August 2010, abgerufen am 08

September 2020. URL : http://journals.openedition.org/sjep/518 ; DOI : https://doi.org/10.4000/sjep. 518

(c) The Graduate Institute 


\title{
Aussichten auf virtuellen Wohlstand
}

\author{
Marie Thorndahl*
}

\begin{abstract}
Die Informations- und Kommunikationstechnologien (IKT) haben in der Diskussion um das Gemeinwohl eine wesentliche Bedeutung gewonnen. Das Versprechen des materiellen Überflusses - an Information, Freizeitbeschäftigung und Wissen - prägt die Entwicklungsdiskussion und bringt neue Konzepte wie „digitale Kluft“, „E-Readiness“, „E-velopment“ usw. hervor. Diese neuen Perspektiven bedeuten einen Jungbrunnen für die internationale Entwicklungszusammenarbeit. Ein „gutes Leben für alle“ scheint greifbar nahe, vorausgesetzt, dass die armen Länder in allen traditionellen Entwicklungsbereichen - Wirtschaft, Gesundheitswesen, Erziehung, Politik, Umweltpolitik usw. - die IKT einsetzen.
\end{abstract}

Diese Hoffnungen werden von Drohungen gegen die Fortschrittsskeptiker begleitet: Nicht angeschlossen zu sein bedeutet zwangsläufig, am Rande der als wohlhabend und demokratisch versprochenen „globalen Informationsgesellschaft" zu bleiben. Das Internet entwickelt sich vom Kommunikationsinstrument zu einem neuen Grundbedürfnis, das dringend befriedigt werden muss. Die Integration der IKT in die Entwicklungsdiskussion erweist sich als ausgezeichneter Träger, um die überholt geglaubten evolutionsbestimmten und technikorientierten Visionen wieder zu verwerten.

Um zu erkennen, was wirklich neu und was weniger neu ist, müssen die IKT vor dem Hintergrund der bisherigen Entwicklungszusammenarbeit betrachtet werden. Die besondere Betonung der IKT verdrängt bisweilen die Tatsache, dass der Technologietransfer bereits seit geraumer Zeit als Vektor für die Entwicklung der südlichen Länder dient - was keineswegs bedeutet, dass die Geschichte ins Stocken geraten ist. Weshalb haben die IKT für die Entwicklungszusammenarbeit so stark an Bedeutung gewonnen? Welchen Einfluss übt die Zusammenarbeit auf die Geografie, die Inhalte und die Nutzung der elektronischen Netze aus? Welche Werte und Interessen werden von den im Namen der „Bekämpfung des digitalen Grabens“ durchgeführten Tätigkeiten übermittelt, und wie fügen sich die IKT in das breitere Phänomen der Globalisierung ein? Ist Entwicklung schliesslich überhaupt noch ein Anliegen, zumal die gesamte Weltbevölkerung eine „globale Informationsgesellschaft“ bilden wird?

Die Informationsgesellschaft bedeutet zunächst eine Chance für die internationalen Organisationen, die darin neuen Enthusiasmus schöpfen und eine neuе Legitimität für ihr Handeln finden. Allerdings dürfte diese Erneuerungsphase nur von kurzer Dauer sein. Noch auffallender als der digitale Bruch ist nämlich das Gefälle zwischen dem Diskurs, der auf die ausserordentlichen Auswirkungen der IKT für möglichst zahlreiche Menschen setzt, und der Realität dieser

* Sozioökonomin, Frankreich. 
Technologien, die nach wie vor nach stark kommerziellen und ungerechten Kriterien konzipiert werden.

Die internationale Zusammenarbeit trägt sowohl passiv als auch aktiv zu diesen Tendenzen bei. Häufig verwandelt sie sich - ohne wirkliche Einflussnahme auf die Netze und von altruistischen Diskursen verdeckt - zur Wortführerin der Interessen der Privatunternehmen: Das Heil liege einzig in den öffentlich-privaten Partnerschaften und in der Öffnung der Telekommunikationsmärkte in den Ländern des Südens. Die Fehlschläge in der New Economy und die Bankrottfälle an der Börse werden geleugnet, damit der Mythos einer Interessengemeinschaft zwischen Info-Reichen und Info-Armen am bald geteilten Wohlstand weiter leben kann.

\begin{abstract}
Comment résister au sentiment ordinaire qui consiste à se penser toujours à un tournant de l'histoire?

(Wie kann man sich gegen das Gefühl wehren, sich immer gerade an einem Wendepunkt der Geschichte zu wähnen?)
\end{abstract}

Gilbert Rist

Nicht oder nur oberflächlich ans Internet angeschlossen zu sein, läuft auf die Ausgrenzung aus dem planetaren Netzsystem hinaus. Das Internet ist heute für die Entwicklung genauso wichtig, wie es die Elektrizität für die Industrialisierung war. In zahllosen Erklärungen wird dafür plädiert, die „echten Probleme der Dritten Welt" - sprich Gesundheit, Ausbildung, Wasser, Elektrizität usw. vor dem Thema Internet anzupacken, was einen grundlegenden Mangel an Verständnis für die aktuellen Probleme der Entwicklung beweist ${ }^{2}$.

Der Soziologe Manuel Castells übt anscheinend harsche Kritik: Die Institutionen, die sich für die Förderung von Sektoren einsetzen, die seit langem als wesentlich für die menschliche Entwicklung gelten, und diesen Zeit, Geld und Fachkompetenzen widmen, sollen mit dem Aufkommen des Internets überholt sein.

Ein kurzer Überblick über die Reden der Vereinten Nationen zeigt jedoch, dass die oben stehende Äusserung nur zum Schein Kritik beinhaltet, und im Gegenteil Wasser auf die Mühlen der internationalen Zusammenarbeit leitet. UNOGeneralsekretär Kofi Annan sagt ja im Grunde dasselbe, wenn er die Informations- und Kommunikationstechnologien (IKT) als eine mächtige Kraft bezeichnet, die für die globale Friedens- und Entwicklungsmission mobilisiert werden muss. Es handelt sich um eine ethische und ökonomische Frage. Die neue Wirtschaft ist langfristig nur dann produktiv und nachhaltig, wenn sie sich in der Welt ausbreitet und die Bedürfnisse und die Nachfrage der gesamten Bevölkerung deckt ${ }^{3}$.

Gilbert Rist, Le développement, histoire d'une croyance occidentale, Presses de Sciences Po, Paris, 1996, S. 344-345.

2 Manuel Castells, La galaxie Internet, Fayard, Paris, 2001, S. 326.

3 Kofi Annan's IT Challenge to Silicon Valley, 5. November $2002<$ http://news.com.com/2010-1071978578.html?tag=rc_spec $>$. 
Angesichts der verschärften Ungleichheiten in der Welt ist zu begrüssen, dass die Staaten - indem sie die internationalen Entwicklungsinstitutionen entsprechend beauftragen - die neuen Technologien in den Dienst der Grundsätze der Menschenwürde, der Gleichheit und der Gerechtigkeit stellen möchten ${ }^{4}$.

Das Internet und die digitalen Informationstechnologien generell ermöglichen das Bearbeiten und Speichern riesiger Informationsmengen und die äusserst schnelle Übermittlung über die elektronischen $\mathrm{Netze}^{5}$. Weshalb und wie sollen diese Technologien die globale Solidarität stärken?

Die internationale Zusammenarbeit beruht auf der Hoffnung, allen Völkern der Welt materiellen Wohlstand zu bringen. Warum und wie kann die Zusammenarbeit dann immaterielle Werte - Information und Kommunikation - beeinflussen? Wie lassen sich die Strukturen und Ideale, die auf der „Entwicklungsära“ aufgebaut sind, an die grundlegend neuen Herausforderungen der „Informationsära" anpassen?

\section{Technik, Werte und Interessen}

Das Konzept der „Informationsgesellschaft“ geht auf die 60er-Jahre zurück Insbesondere ab Ende der 80er-Jahre hat es sich in Studien, Veröffentlichungen und amtlichen Berichten eingebürgert und in der Soziologie als Paradigma etabliert. Für Verfechter und Gegner gilt jedoch, - ungeachtet der unklaren Grundlagen - dass die Informationsgesellschaft in erster Linie im Diskurs und in den Analysen existiert, zu denen sie Anlass gibt.

Ein wichtiges Merkmal der Informationsgesellschaft bildet die Annahme, dass die Gesellschaft und die Wirtschaft um die IKT herum strukturiert sind. Zur Diskussion steht dagegen der Grad des technischen Determinismus bzw. des sozialen Einflusses der neuen Technologien ${ }^{7}$. Evolutionistische Kreise sehen die IKT als Zeichen des Übergangs zu einer neuen wirtschaftlichen Entwicklungsphase (Landwirtschaft - Industrie - Information), wobei die IKT per se zwar nicht die sozialen Beziehungen verändern, aber die Produktion und die Verteilung des Wohlstands in der Welt bestimmen - keine Bagatelle also. Andere,

4 Vereinte Nationen, von der Generalversammlung verabschiedete Resolution, Déclaration du Millénaire, New York, 8. September 2000.

5 Der Sektor der Informations- und Kommunikationstechnologien umfasst sehr unterschiedliche, auf den wissenschaftlichen Fortschritten in der Informatik, Elektronik, Optik, Software, Satellitentechnologie usw. gegründete Systeme und Dienstleistungen. Sichtbarster Ausdruck der „Revolution“ ist das Internet, bei dem sich Informatik und Telekommunikation verbinden. Die Konvergenz erlaubt es, riesige Informationsmengen zu bearbeiten, zu speichern und in Hochgeschwindigkeit über die elektronischen Netze zu übermitteln. Die Speicher- und Übertragungskapazitäten wachsen bei sinkenden Kosten exponentiell an. Siehe Tabelle in UNDP, Rapport Mondial sur le développement humain 2001, Mettre les nouvelles technologies au service du développement, New York, Oxford, 2001, S. 32.

6 Die Herkunft des Konzeptes - Japan oder die Vereinigten Staaten - ist umstritten. Für nähere Auskünfte über die Entstehungsgeschichte wird verwiesen auf: Alistair S. Duff, Information Society Studies, Routledge, London \& New York, 2000, S. 2-6; und Armand Mattelard, Histoire de la société de l'information, La découverte, Paris, 2001.

7 Zur Vertiefung der Diskussion siehe Alistair S. Duff, ibid. 
radikalere Kreise vertreten die Auffassung, dass die IKT grundlegende Umwälzungen in der Gesellschaft und in den sozialen Institutionen auslösen ${ }^{8}$. Diese Revolution wird generell als Tendenz zu grösserem sozialem Fortschritt bewertet $^{9}$. Die Diskussion ist geprägt von Assoziationen mit dem „wiedergefundenem Paradies auf Erden“ - dank den Segnungen der Kommunikation und selbst um den Preis, die alten Strukturen aufzulösen, um Platz für das Neue zu schaffen.

Wir wollen uns hier nicht mit dem Wesen der "Informationsgesellschaft“ beschäftigen. Stattdessen gehen wir vom Postulat aus, dass die IKT nicht aus heiterem Himmel fallen, sondern dass sie eine Geschichte haben, vor dem Hintergrund sozialer Beziehungen betrachtet werden müssen und keine „Einbahnstrasse" bedeuten: Die IKT können zentrale oder dezentrale Kräfte freisetzen, Teil des demokratischen Kontextes bilden oder aber ein autoritäres Instrument sein, Reichtum bringen oder hohe Kosten verursachen.

Ausserdem dürfen die der Information und Kommunikation zu Grunde liegenden Werte nicht vergessen werden. Es handelt sich um Werte, die wie die Freiheit und die Brüderlichkeit tief in der kulturellen und politischen Tradition des Westens verwurzelt sind - starke und generell deutlich positiv konnotierte Werte. Im Zuge der explosionsartigen technischen Entwicklung hat die technologische Dimension die menschliche Dimension verdrängt. Die Verfechter der Technik führen die Kommunikation in die Ära der Profite ein. Die Logik der Werte prallt auf die Logik der Interessen: Der Unterschied ist entscheidend wichtig, zumal das Instrumentalisieren der Kommunikation, als Folge der leistungsfähigen Technik und der Öffnung grosser Märkte, die Akteure der Politik und der Industrie davon träumen lässt, beide Aspekte zu vermischen bzw. die Gesellschaft von morgen schlicht und einfach mit den vorherrschenden Techniken gleichzusetzen ${ }^{10}$.

\section{Entwicklung der anderen}

Der Glaube an die Verallgemeinerung der „Entwicklung“ hat zwar gelitten, aber der altruistische Diskurs ist auch im Zeitalter der Globalisierung noch präsent. Zahlreiche Akteure und Einrichtungen verfolgen nach wie vor das Ziel, durch die Wohltaten der Entwicklung, diesmal dank des von der Informationsgesellschaft verheissenen materiellen Wohlstandes, ,ein gutes Leben für alle“11 $\mathrm{zu}$ ermöglichen. In der Folge werden die internationalen Organisationen der Entwicklungszusammenarbeit darauf hin geprüft, wie diejenigen, welche von der Entwicklung der anderen leben und dazu beträchtliche materielle und symbolische Ressourcen mobilisieren ${ }^{12}$, die Umwälzungen und die Versprechen der Informationsgesellschaft mit einbeziehen.

8 Zu den Autoren, welche diese Position verfechten, gehören Manuel Castells, op. cit. oder Joël de Rosnay, L'Homme symbiotique. Regards sur le troisième millénaire, Le Seuil, Paris, 1995.

9 Mattelard, op. cit., S. 59. Selbstverständlich wird die Entwicklung auch von anderen kritisiert, darunter Dominique Wolton oder Philippe Breton, Le culte de l'Internet: une menace pour le lien social?, La Découverte, Paris, 2000.

10 Dominique Wolton, Internet et après?, Flammarion, Paris, 1999, S.11.

11 Gilbert Rist, op. cit., S. 349.

12 Jean Pierre Olivier de Sardan, Anthropologie et développement, Karthala, Paris, 1995, S. 7. 
Jean-Pierre Olivier de Sardan definierte im Jahr 1995 im Kontext der ländlichen Entwicklung in Afrika die Entwicklungskonfiguration als weitgehend kosmopolitisches Universum von Experten, Bürokraten, NGO-Verantwortlichen, Forschern, Technikern, Projektleitern und Ausführenden vor Ort ${ }^{13}$. Welche Akteure produzieren im Bereich der IKT die Theorie, und welche die konkreten Resultate? Wer setzt sie um, und wozu?

\section{Diskurs: Neue Bedürfnisse, neue Versprechen}

Die Informationsgesellschaft bildet die positive Seite einer sich neu gebenden sozioökonomischen Organisation, während die digitale Kluft die Kehrseite der Medaille darstellt - das Zeichen des neuen Ausschlusses, den sie hervorruft, und der neuen, noch nicht konkretisierten Bedürfnisse.

Angesichts der Asymmetrie im Zugang und/oder in der Nutzung der digitalen Technologien lässt sich der Begriff „,digitale Kluft“ mit einer breiten Palette von Bereichen kombinieren: Geografie - Unterschiede im Zugang nach Regionen, zwischen Ländern und Gebieten des gleichen Landes; Demografie - Unterschiede zwischen Altersgruppen; Kultur - Unterschiede zwischen soziokulturellen, ethnischen oder sprachlichen Gruppen usw.; Ökonomie - Betonung der Unterschiede des Zugangs nach Einkommensschichten und Entwicklungsstand. Zusammengefasst dient die digitale Kluft als Sammelbegriff für die Beschreibung der mit der Ausdehnung der IKT zusammenhängenden Ungleichheiten.

Die Fachliteratur über die digitale Kluft beruht in der Regel auf schwachen theoretischen Grundlagen und auf dürftiger Feldforschung; bestimmend ist die Intuition der Autoren: Die meisten Forschungsarbeiten sind prospektiv, optimistisch und handlungsorientiert ${ }^{14}$. Die Analyse der Verbindungen zwischen Technologiezugang, sozialem Fortschritt und Wirtschaftswachstum bleibt lückenhaft. So wurde zwar die Korrelation zwischen der Anzahl Computer mit Internetanschluss und der Einkommenshöhe nachgewiesen, doch kann nicht mit Gewissheit gesagt werden, ob die Computer den Wohlstand herbeigeführt hätten oder ob der Wohlstand die Ausstattung mit Computern erlaubt habe ${ }^{15}$.

Deshalb beschränken sich die meisten Berichte auf die quantitative lineare Relation zwischen den „Habenden“ und den „Habenichtsen“ und ignorieren die Vielfalt der Situationen. Der Akzent liegt auf dem, was die Industrieländer besitzen und was den Entwicklungsländern fehlt: Technologie, Infrastruktur, Wissen und technisches Know-how. Angeprangert wird nie der Informationsüberfluss im Norden, sondern das Informationsdefizit im Süden, obwohl es zur Entstehung des Problems ja beide braucht - die „Info-Reichen“ und die „Info-Armen“. Aus der Feststellung eines Gefälles wird eine neue - immaterielle - Armut und

13 Jean Pierre Olivier de Sardan, ibid.

14 Die Literatur stammt im Wesentlichen von den Organisationen, welche Programme zur Behebung des digitalen Grabens durchführen. Deshalb werden die getroffenen Massnahmen logischerweise gerechtfertigt.

15 Institute for Prospective Technological Studies, The Digital Divide: A Research Perspective, Report to the G8 Digital Opportunities Task Force, Sevilla, 2001. 
eine Aufforderung zum Handeln abgeleitet: Der Internetanschluss ist ein „Must“. Nach dem neuen Paradigma der Informationsgesellschaft wären andere Politiken durchweg unvernünftig.

\section{Enthusiasmus und Jungbrunnen für die Entwicklung}

Die „digitale Kluft“ ist ein besonders beliebtes Konzept der internationalen Zusammenarbeit. Den Bruch zu kitten und die IKT in den Dienst der Entwicklung zu stellen, sind seit einigen Jahren vorrangige Anliegen der „Entwicklungsagenden" und globale Ziele der weltweiten politischen Führungskreise geworden ${ }^{16}$. Die Millenniumserklärung der Vereinten Nationen gibt mit folgendem Ziel den Ton an: ...dafür sorgen, dass alle in den Genuss der Vorteile der neuen Technologien, insbesondere der Informations- und Kommunikationstechnologien, kommen [...] die IKT können sich als mächtige Werkzeuge erweisen, um das allgemeine Wachstum und die nachhaltige Entwicklung zu beschleunigen und die Armut zu bekämpfen. Wenn ganze Regionen der Welt einen Rückstand in der Konnektivität und im Zugang zu den globalen Informations- und Wissensnetzen aufweisen, werden sie aus der auf Wissen basierenden Weltwirtschaft, die sich heranbildet, ausgegrenz $t^{17}$. Die IKT werden auf alle Bereiche angewandt - Gesundheit, Erziehung, Arbeit, Umwelt, Gleichstellung der Geschlechter usw. In den verschiedenen Jahresberichten beleuchten die Organisationen die Herausforderungen der digitalen Kluft aus ihrer spezifischen Warte ${ }^{18}$.

Diejenigen, welche die „Entwicklung“ und ihre Institutionen als Relikte des 20. Jahrhunderts abtaten, sehen es ungern, dass die Integration der IKT in die allgemeine Entwicklungsproblematik offensichtlich einen Jungbrunnen für die internationalen Institutionen bedeutet. Dass die Vereinten Nationen sich mit der digitalen Kluft beschäftigen, ist indessen noch nicht als Wendepunkt der Geschichte zu deuten. Gegen den digitalen Graben vorzugehen, ist unumstritten. Gleiches gilt für den Umweltbereich. All dies bedeutet jedoch noch kein strukturelles Umdenken in der Problematik.

\section{Viele Akteure, eine Devise}

Die Herausforderung liegt anderswo: bei der Zunahme der Partnerschaften zwischen Staaten, Industrien, internationalen Finanzinstitutionen, Entwicklungsorganisationen, Forschungszentren, Organen der Vereinten Nationen usw., welche für eine tief greifende Umgestaltung der Landschaft der internationalen

16 UNDP, op. cit., S. 38.

17 Vereinte Nationen, Generalversammlung, Plan de campagne pour la mise en æuvre de la Déclaration du Millénaire, Rapport du Secrétaire général, 6. September 2001.

18 Die vier wichtigsten sind Folgende: Die UNESCO veröffentlicht 1999 den Rapport mondial sur la Communication et l'Information 1999-2000 über die Nutzung der Informationstechnologien in den Bereichen Erziehung, Kultur und Kommunikation - UNESCO, Rapport mondial sur la communication et l'information 1999-2000, Paris, 1999. Im Jahr 2000 publiziert das UNDP den Rapport Mondial sur le développement humain 2001, Mettre les nouvelles technologies au service du développement - UNDP, op. cit. Im Jahr 2002 legt die Internationale Arbeitsorganisation den Rapport sur l'emploi dans le monde 2001. Vie au travail et économie de l'information vor - BIT, Rapport sur l'emploi dans le monde 2001. Vie au travail et économie de l'information, Genf, 2002 - UNCTAD, E-commerce and Development Report 2002, New York, Genf, 2002. 
Zusammenarbeit bezeichnend ist. Kein Programm wird im IKT-Bereich mehr ohne die Betonung der Triade „Staat-Wirtschaft-Zivilgesellschaft“ durchgeführt. Die IKT zu beeinflussen bedeutet auch, sich an die Speerspitze der Einführung neuer Typen der Global Governance zu stellen.

Nachstehend werden aus der beeindruckenden Anzahl internationaler Initiativen, die innerhalb weniger Jahre zu diesem Thema ${ }^{19}$ gestartet wurden, die wichtigsten vorgestellt.

Die Weltbank - Pionier im Bereich IKT für die Entwicklung - bildet einen unumgänglichen institutionellen und finanziellen Angelpunkt. Bereits 1995 startete sie das Programm Information for Development, in welchem sie den Privatsektor auffordert, sich an vorderster Front für Initiativen einzusetzen, welche ergriffen werden, um - über den Ausbau der Informationsinfrastrukturen die Rolle der IKT zu stärken ${ }^{20}$. Seitdem hat die Weltbank zahlreiche auf öffentlich-privaten Partnerschaften beruhende Programme lanciert, darunter auch den Development Gateway ${ }^{21}$, der die Inhalte auf die Entwicklung ausrichten soll. Anlässlich der PrepCom I zum Weltgipfel über die Informationsgesellschaft (WSIS) äusserte sich der Weltbankvertreter wie folgt: In den letzten Jahren hat die Weltbankgruppe für diesen Sektor Darlehen im Wert von durchschnittlich 2,5 Milliarden Dollar (IBRD), Investitionen von 500 Millionen Dollar (IFC) und Schenkungen in Höhe von knapp 30 Millionen gewährt. Damit ist sie bei weitem der wichtigste Akteur, was die IKT im Dienste der Entwicklung anbelangt $t^{22}$.

Zu den herausragenden Initiativen zählt auch die im Jahr 2000 vom G-8 eingesetzte Digital Opportunity Task Force, die aus Vertretern der Staaten, der Industrie und der Zivilgesellschaft besteht. Die hauptsächlich unter der Bezeichnung DotForce bekannte Expertengruppe hat einen Aktionsplan zum Umgang mit den Herausforderungen der digitalen Kluft ${ }^{23}$ ausgearbeitet. Der Aktionsplan wurde anlässlich des Gipfels von Genua im Jahr 2001 bestätigt.

Eine weitere wichtige Initiative ist die UN-ICT Task Force ${ }^{24}$, die im Jahr 2000 als innovatives Instrument zur Steuerung der Politik der Vereinten Nationen im IKTBereich lanciert wurde. Die Mitglieder - Vertreter von Regierungen, des Privatsektors, der Weltbank, von Entwicklungsorganisationen, Universitäten und Organisationen der Vereinten Nationen - sollen folgende komplexe Frage beantworten: Wie können die IKT die digitale Kluft überbrücken, um die Entwicklung hinsichtlich der Ziele des Millenniumsgipfels - ausgehend von Partnerschaften zwischen zahlreichen Akteuren des öffentlichen und privaten Sektors -

19 Im November 2000 listete das Sekretariat der DotForce über 40 verschiedene Initiativen auf. Finance Canada, The Digital Divide: the Challenge of Coordination, Februar 2001, <www.bellanet.org/ dotforce/docs/Fifth_Paper_Final.doc?ois=y;template=blank.htm $>$.

20 Banque Mondiale, Technologies de l'Information et des Communications: Stratégie du Groupe Banque Mondiale, <http://info.worldbank.org/ict/>.

$21<$ www.developmentgateway.org $>$.

22 Genf, Juli 2002, <www.wsis.org >.

23 „Digital Opportunities for All: Meeting the Challenge“, <www.dotforce.org/>

24 Auf die Initiative des Wirtschafts- und Sozialrates (ECOSOC) eingesetzt: Französisch „Groupe d'étude sur les technologies de l'information et de la communication“, <http://www.unicttaskforce.org/> 
Beim Global Knowledge Partnership ${ }^{26}$ (bis 2003 unter dem Vorsitz der schweizerischen Direktion für Entwicklung und Zusammenarbeit, DEZA), handelt es sich ebenfalls um ein Netz des Typs ,Staat-Wirtschaft-Zivilgesellschaft-internationale Finanzinstitutionen“, welches den Zugang und den Einsatz der IKT für die Entwicklung vorantreiben soll.

$\mathrm{Zu}$ den globalen Initiativen kommen zahlreiche, durchweg auf dreigliedrige Partnerschaften abgestützte sektorenbezogene Programme hinzu; darunter die von der Internationalen Fernmeldeunion (ITU) im Jahr 1997 gegründete World$\mathrm{Tel}$. Dabei handelt es sich um eine Aktiengesellschaft von Grossunternehmen, welche Finanzierungen zur Behebung des Gefälles im Kommunikationsbereich finden soll ${ }^{27}$. Auch die vom United Nations Development Program (UNDP) mit der Markle Foundation und der Managementberatungsfirma Accenture durchgeführte Digital Opportunity Initiative ${ }^{28}$ zielt darauf ab, öffentlich-private Projekte im Bereich der Informationstechnologien zu fördern. Die vom Weltwirtschaftsforum in Davos im Jahr 2000 gestartete Global Digital Divide Initiative ${ }^{29}$ umfasst ebenfalls Grossunternehmen, Organisationen wie die Weltbank, die Internationale Fernmeldeunion (ITU) und die Harvard-Universität. Microsoft und das Centre de recherche sur le développement (CRDI) der kanadischen Behörde für Entwicklungszusammenarbeit haben gemeinsam den Vorsitz inne.

Diese und viele weitere Initiativen müssen anlässlich des von den Vereinten Nationen organisierten Weltgipfels über die Informationsgesellschaft (WSIS) harmonisiert werden. Der Gipfel verfolgt auch das Ziel, die Vorteile der Informationsgesellschaft in die Reichweite aller zu rücken ... und die digitale Kluft zu überwinden ${ }^{30}$.

\section{Die Business-Philosophie}

Diese neue Entwicklungszusammenarbeit gibt sich demokratischer und realitätsnäher. Wer sind denn aber die „,wirklichen“ Akteure der Informationsgesellschaft? Ist nicht der Privatsektor der wichtigste Konstrukteur, Verwalter und Eigentümer von Kommunikationsnetzen? Oder was wäre die Informationsgesellschaft ohne die Zivilgesellschaft, die Hauptnutzerin der Netze? Die neuen Partnerschaften scheinen vielversprechend ${ }^{31}$, aber was leisten sie wirklich?

In erster Linie produzieren sie Diskurse. Gemeinsames Leitmotiv ist die Erbringung des Nachweises, dass der digitale Graben im Grunde eine Chance darstellt, und dass sich die Interessen zwischen dem Streben nach privatem Profit

\footnotetext{
$<$ www.un.org/french/ga/56/ticag.htm>.

$<$ www.globalknowledge.org/>.

$<$ www.world-tel.com/ $>$.

$<$ www.opt-init.org/>.

<www.weforum.org/site/homepublic.nsf/Content/Global+Digital+Divide+Initiative >.

Bericht des Unterausschusses II thèmes et contenus, PrepCom 1, Juli 2002, <www.wsis.org>.

Bestimmt vielversprechender, als wenn das gleiche Schema auf Bereiche wie Erziehung oder Umgang mit natürlichen Ressourcen - die traditionell nach den Prinzipien der öffentlichen Dienstleistung verwaltet werden - angewandt wird.
} 
und möglichst generellem Wohlstand decken. Die lineare Demonstration bedient sich dabei gängiger Vorstellungen:

$\square$ Beunruhigung wegen der abgrundtiefen ,digitalen Kluft“, dem gravierenden „digitalen Bruch“, der „digitalen Apartheid“ oder des Ausmasses der „Informationswüste“".

$\square$ Genugtuung über das Potenzial der IKT zur Behebung der Entwicklungsprobleme, zum Einlösen der Versprechen des „digitalen Frühlings“, der „digitalen Renaissance“, der „digitalen Revolution“ als „Startrampe für die Entwicklung“"

- Schlussfolgerung: Die neue Chance für die Armen ist mit den Interessen der Geschäftswelt vereinbar: Indem die Geschäftswelt die akuten Bedürfnisse der Armen befriedigt, leistet sie gleichzeitig für die ganze Menschheit Gutes. Dabei ist die Rede von ,digitalen Dividenden“" und „digitalen Chancen“, die Profite und soziale Gewinne erzeugen.

Die Stellungnahmen des Wirtschafts- und Sozialrates (ECOSOC) und des Generalsekretärs der Vereinten Nationen sollen - selbst wenn es noch enthusiastischere Diskurse gibt - als Veranschaulichung dieser Logik ausreichen. Zur Begründung der Wichtigkeit der UNICT-Task-Force stellt der ECOSOC den Ländern des Südens einen ,elektronischen Frühling“ in Aussicht: Die am wenigsten entwickelten Länder werden zwar im Bereich der Hochtechnologie benachteiligt, doch kann die elektronische Revolution sich als Startrampe für die Entwicklung erweisen. Die Kapazitäten der IKT können die kleinen und mittleren Unternehmen der aufstrebenden Volkswirtschaften und sogar die lokalen Handwerker der ärmsten und entlegensten Länder mitten in die nationalen, regionalen und globalen Märkte katapultieren. Die IKT setzen sich über die
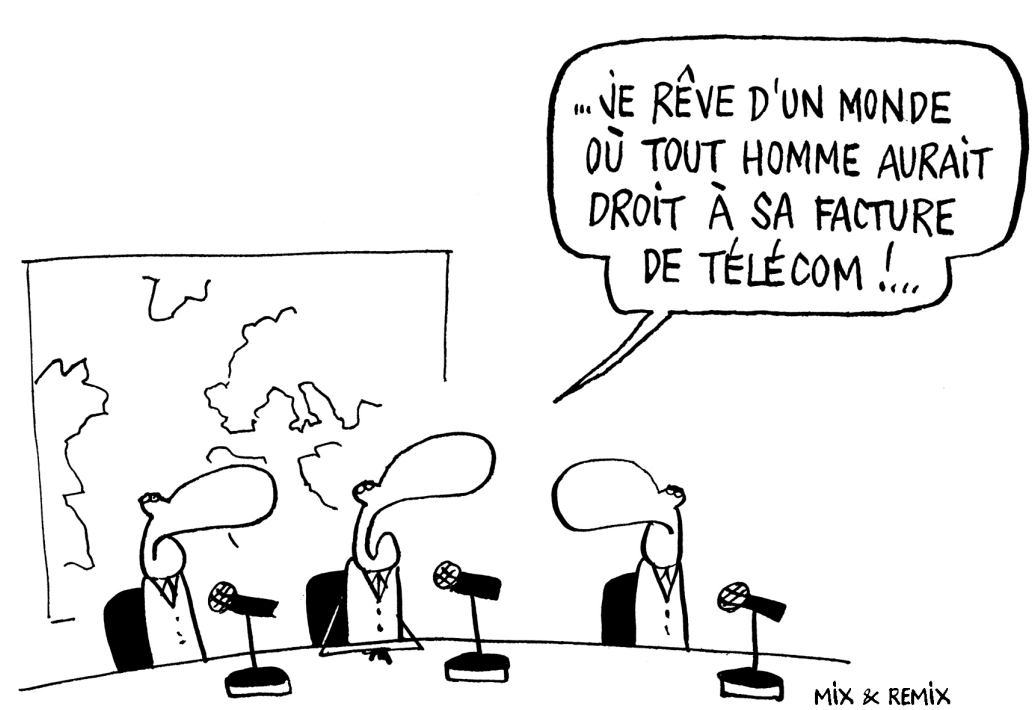

Ich träume von einer Welt, in der alle Menschen Recht auf eine Telekomrechnung haben... 
Hindernisse der Entwicklung hinweg; sie verhüten Katastrophen und wirken Wunder, wie z.B. bei auf Distanz durchgeführten medizinischen Operationen ${ }^{32}$. Dazu hält der Generalsekretär der Vereinten Nationen folgendes Plädoyer: Die Unterstützung des Privatsektors spielt eine besondere Rolle. Die Nutzung des Internets für die Entwicklung gehört glücklicherweise zu den Bereichen, in denen die langfristigen Interessen der internationalen Staatengemeinschaft, der Regierungen und der Geschäftswelt eindeutig aufeinander treffen. Das Empowerment der Armen und Ausgegrenzten kann kreative Energien freisetzen, die den Spielraum für kleine und mittlere Unternehmen vergrössern helfen. Sie können dazu beitragen, Märkte zu schaffen und zu erweitern. Privatunternehmen helfen sich selbst, indem sie anderen helfen ${ }^{33}$.

Die Staaten des Südens und des Ostens werden aufgefordert, die E-Readiness ein Gradmesser für die Integrationsfähigkeit in die globale Informationswirtschaft - durch die Einführung von „E-Strategien“ zu steigern: Nur diese können die exponentielle Kurve des E-veloppement auslösen ${ }^{34}$. Dazu müssen insbesondere das E-Leadership (d.h. die politische Priorität, die der Ausbreitung der IKT beigemessen wird), die Good Governance und die "Qualität der öffentlich-privaten Partnerschaften“ sowie das Rechts- und das Regelungsumfeld verbessert werden, um den E-Commerce, die Systemsicherheit und die Konnektivität zu entwickeln.

Auf der Basis der von amerikanischen Managementfirmen ${ }^{35}$ ausgearbeiteten Indikatoren wird jährlich ein Ranking der Länder durchgeführt. 2002 waren die Vereinigten Staaten in Sachen E-Readiness Klassenbeste. Die Schweiz lag auf Platz vier. Die Schlusslichter bildeten Algerien, Kasachstan und Aserbaidschan. Unter den Ländern südlich der Sahara hatten nur Südafrika und Nigeria, welche die 35. bzw. die 56. Stelle von 60 einnahmen, überhaupt die Ehre, im Rennen dabei zu $\operatorname{sein}^{36}$. Der Indikator wird auch von den internationalen Institutionen mit einem altruistischen Anstrich versehen - aufgegriffen. Auf die Frage: Warum wollen die Länder „e-ready“ sein?, antwortet die Weltbank: Die IKT sind eine Schlüsselwaffe im Kampf gegen die Armut ${ }^{37}$. Geschickter Trick: Die von der Geschäftswelt für die reichen Länder konzipierten Kriterien werden

3 Vereinte Nationen, Pressemitteilung, 20. November 2001, <www.unicttaskforce.org/welcome/principal. asp>.

34 Im Gegensatz zur Kurve der traditionellen Entwicklung, die ein quasi lineares sozio-ökonomisches Wachstum aufweist. Bruno Lanvin, „La fracture numérique n'est pas une fatalité“ in Nord et Sud numériques, unter der Leitung von Jean-François Soupizet und Laurent Gille, Hermes Science Publication, Paris, 2002, S.32.

$35<$ <ww.mcconnellinternational.com/> - Für die Anwendung auf den Fall der Länder des Südens wird insbesondere verwiesen auf Martin R. Hilbert, Latin America on its path into the digital age: where are we? CEPAL, Chile, 2001, S.36-37.

$36<$ www.ebusinessforum.com/ $>$.

37 Weltbank, E-Readiness as a tool for ICT development, November 2001 - <www.infodev.org/ library/WorkingPapers/AReready.pdf $>$. Seitdem finanziert die Weltbank auch das E-Readiness centre $<$ www.ereadinesscenter.org/>. Das UNDP hat in Zusammenarbeit mit den Universitäten und dem Privatsektor ein eigenes Netz zum Thema geschaffen, um die Länder zu den umzusetzenden E-Strategien zu beraten. Für eine Liste der Methoden betreffend die E-Readiness siehe <www.infodev. org/ereadiness/methodology.htm>. 
zum Massstab, den die ärmsten Länder - in ihrem eigenen Interesse ! - einhalten müssen und ,wollen“.

Einerseits besteht Macht nicht unbedingt darin, die Wirklichkeit zu verändern, sondern sie anders zu problematisieren, d.h. neu darzustellen und damit die Illusion von Veränderungen zu wecken ${ }^{38}$; andererseits hat sich das BusinessDenken, das die meisten internationalen Gremien prägt, fest eingebürgert. Damit wird die Logik der Interessen und der Werte durcheinander gebracht und der Handlungsspielraum eingeengt - Ja und Amen! Die Ausdehnung der IKT muss auf Marktstrategien beruhen. Die internationalen Organisationen, die Studiengruppen und die auf IKT spezialisierten akademischen Forschungsgruppen übernehmen diese Theorien und fördern diese Entwicklung. Damit erteilen sie der Legitimität des Marktes den internationalen Segen ${ }^{39}$ und bieten ausserdem ihre materiellen und menschlichen Ressourcen an. Die Dreigliedrigkeit wird zu einer Inszenierung des Konsenses, der das bestehende Kräfteverhältnis getreulich abbildet.

\section{Öffentliche Ressourcen schwinden}

Die internationale Zusammenarbeit im Bereich der Telekommunikation beruht demnach auf folgendem Paradigma:

$\square$ Infrastrukturen spielen für die Entwicklung eine wesentliche Rolle, aber Installation und Wartung sind sehr kostspielig;

$\square$ es braucht private Finanzierungen;

$\square$ um Privatinvestoren anzuziehen, müssen die Entwicklungsländer ihre Märkte liberalisieren und ein für ausländische Firmen attraktives Regelungssystem einführen.

Die Hauptaufgabe der internationalen Organisationen besteht darin, die Staaten beim Aufbau eines attraktiven Regelungsrahmens zu unterstützen, welcher den Anforderungen der Privatunternehmen entspricht. Die Telekommunikation muss zu einem rentablen Geschäft werden; anderenfalls drohen alle erwähnten Strategien ins Leere zu laufen. Das Eigentum der Netze geht vom öffentlichen an den privaten Sektor über, damit Wettbewerb entsteht, die Preise sinken und indem die Telekommunikation den Ärmsten zugänglich gemacht wird - die Nachfrage steigt. Im Prinzip wird die „Marktliberalisierung“ mit dem ,universellen Zugang“" kombiniert und dabei der Eindruck erweckt, dass diese Aspekte sich systematisch ergänzen und gegenseitig stärken.

Zwischen 1984 und 1999 wurden hundert Telekommunikationsunternehmen teilweise oder ganz privatisiert ${ }^{40}$. Die Weltbank beurteilt die Ergebnisse ihrer Programme als beeindruckend. In über 88 Ländern weltweit sind die Betreiber privatisiert worden, über 63 Länder haben zur Unterstützung des Reformpro-

38 Gilbert Rist, op. cit., S. 130.

39 Gilbert Rist, op. cit., S. 363.

40 Winseck Dwayne, ,The WTO, Emerging Policy Regimes and the political Economy of Transnational Communication“ in Raboy Marc (ed.), Global media Policy in the New Millennium, University of Luton Press, Luton, 2002, S.24. 
Seit den 90er-Jahren haben internationale Organisationen wie die ITU und das UNDP, gefolgt von zahlreichen öffentlichen und privaten Institutionen, ihre Mandate neu definiert. Anstatt wie in der Vergangenheit beim Aufbau von Infrastrukturen und in der Projektfinanzierung mitzuwirken, konzentrieren sie sich auf die Forschung und Beratung. Die Ausarbeitung und Einführung von nationalen Regelungsrahmen und „E-Strategien“ hat sich zu einem blühenden Geschäft entwickelt. Die dafür ausgegebenen Hilfsbeträge übersteigen mittlerweile die Aufwendungen für die Entwicklung der Netze und für die Dienstleistungen ${ }^{42}$.

Abgesehen davon, dass die internationalen Organisationen durch ihre Beratungstätigkeiten die eigenen Strukturen reproduzieren, verfügen sie nur über knappen Handlungsspielraum. Die öffentliche Hilfe verzeichnet einen Tiefststand $^{43}$. Entgegen allen Verlautbarungen, welche der Kommunikation eine zentrale Stelle einräumen, sind die öffentlichen Finanzierungen für die Entwicklung der Telekommunikation rückläufig ${ }^{44}$.

Dass das account rate system (Telefongebühren-Aufteilungssystem) zu Gunsten der Länder des Nordens geändert wurde, hat die öffentlichen Ressourcen für die Länder des Südens weiter verknappt. Das System diente seit Anfang des 20. Jahrhunderts zur Aufteilung der Telefongesprächskosten auf das Anrufer- bzw.

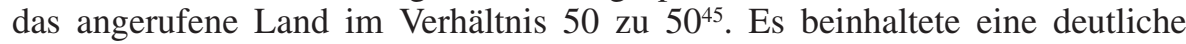
Begünstigung der ärmsten Staaten ${ }^{46}$. Auf Betreiben der amerikanischen Federal Communication Commission (FCC) wurde die Aufteilung der Gebühr aufgehoben. Durch die Privatisierung der Telekommunikationsbetreiber wird das System grundsätzlich in Frage gestellt: Da das Prinzip der Telekommunikation als eine von den Staaten gemeinsam erbrachte, internationale Dienstleistung ausgedient hat, kann keine Gebühr erhoben werden ${ }^{47}$. Diese Veränderung verursacht den afrikanischen Betreibern Verluste von jährlich einer Milliarde Dollar ${ }^{48}$.

Gleichzeitig stellt die Informationstechnologie eine der weltweit mächtigsten Branchen dar und macht ungefähr $10 \%$ der Weltwirtschaft aus ${ }^{49}$. Die Konzentration der Medien-, Informatik- und Telekommunikationsindustrie wird durch

$41<$ http://info.worldbank.org/ict/gictResults.cfm?doc=72>.

42 Winseck Dwayne, op. cit., S. 22-24.

43 Zwischen 1990 und 1999 ist die öffentliche Entwicklungshilfe von 0,34 auf 0,24\% des BSP der Geberländer gesunken. UNDP, op. cit., S. 190 - Auf Privatinvestitionen zu setzen, minimiert selbstverständlich die Verantwortung der Staaten des Nordens, die ihren Verpflichtungen in der öffentlichen Entwicklungshilfe nicht nachkommen.

44 Cees J. Hamelink, Ethic in Cyberspace, Sage Publication, London, 2000, S. 96-97.

$45<$ <ww.itu.int/newsarchive/press/WTPF98/Whatisaccountrate-fr.html>.

46 Laut der ITU stiegen die Nettozahlungsflüsse der Industrieländer in Richtung Entwicklungsländer zwischen 1993 und 1998 auf rund 40 Milliarden USD an. ITU, Réforme du système des taxes de répartition entreprise par la Commission d'études 3 de l'UIT-T, 2002 <www.itu.int/ITU-T/ studygroups/com03/accounting-rate/index-fr.html>.

47 Cees J. Hamelink, op. cit., S. 95-97. In den gleichen Zusammenhang gehört auch die Schuldenkrise, die einen Aderlass für den Staatshaushalt der südlichen Länder bedeutet.

48 Jean-Louis Fullsack, L'UIT la vieille dame des télécommunications dans la tourmente néolibérale 2002, S. $13<w w w . g l o b e n e t . o r g / c s d p t t />$.

49 Verlässliche Zahlen sind Mangelware. Die vorliegende Schätzung stammt von Igniacio Ramonet, Les seigneurs des réseaux, Le Monde Diplomatique, Mai 2002. 
eine Serie von Fusionen der multinationalen Konzerne noch beschleunigt $t^{50}$. Die UNCTAD schätzt die diesbezüglichen Ausgaben für das Rezessionsjahr 2001 auf 988 Milliarden USD. Für die darauf folgenden Jahre geht sie von einem kräftigen Wachstum aus ${ }^{51}$. Zur Finanzmacht kommt die Tatsache, dass sich die meisten Infrastrukturen - Träger des ganzen Kommunikations- und Finanztransaktionssystems - im Besitz des Privatsektors befinden: Kein Wunder also, dass diese Kräfteverhältnisse Politiken herbeiführen, welche die staatliche Intervention beschneiden.

Angesichts der Vormacht der „Business-Philosophie“ und des „techno-liberalen“ Denkens ${ }^{52}$, wonach das Eingreifen des Staates in den freien Markt Tabu ist, haben kreative Mechanismen für die Finanzierung der Ausdehnung des Internets nach einer nicht-kommerziellen Logik schlechte Karten. Gemäss dem UNDP-Jahresbericht, der die mögliche Einführung einer Pauschalgebühr auf den elektronischen Handel erwähnt ${ }^{53}$, drohte der amerikanische Kongress mit einem Beitragsstopp an die Vereinten Nationen, falls diese weitere Vorschläge $z$ Abgaben entwickeln oder fördern, welche die amerikanische Wirtschaft berühren könnten ${ }^{54}$.

Solche Drohungen wurden ernst genommen: In späteren Berichten spricht das UNDP nicht mehr von Abgaben, hält aber fest, dass die Industrie mit ihren Vertretern, welche die Regierungsdelegierten in den Verhandlungen zu den internationalen Übereinkommen begleiten, grossen Einfluss auf die Ausgestaltung von Politiken und Regelungen nimmt. Die Schwergewichte der Industrie begründen einen Status quo in der Art und Weise, auf welche die Regierungen Regeln für die Geschäftswelt erlassen, und dieser Status quo lässt sich selbst dann nur schwer ändern, wenn die Öffentlichkeit weiss, dass etwas „faul“ ist ${ }^{55}$.

\section{Geografie der Netze: Bilanz}

Mit 665 Millionen Internetnutzern (Ende 2002) ${ }^{56}$ sind 90\% der Weltbevölkerung von den Kommunikationsnetzen ausgeschlossen. Kein Wunder: Das Internet wurde auf einer elitären Grundlage strukturiert. Dies ist an sich nicht verwerflich, wirkt sich aber tief greifend auf die Architektur des Internets aus. Das Internet wurde in nordamerikanischen und europäischen Forschungszentren des

50 UNESCO, op. cit., S. 27-38.

51 UNCTAD, op. cit., S. 110.

52 Die vorgeschlagene Steuer auf die Transaktionen wird auch von den Net-Pionieren oder den „Techno-liberalen“, die darin eine unerträgliche Einmischung des Staates zur Bürokratisierung und zur Kontrolle des Cyberspace sehen, vehement bekämpft. Siehe dazu Armand Mattelard, op. cit., S. $98-100$.

53 Vorschlag der „Bit Tax“. UNDP, Human Development Report 1999 - Globalization with a Human Face, Kapitel 5.

54 Prohibition on United Nations Taxation Act of 1999 (Introduced in the House), 106th Congress, 6. Januar, 1999 <www.globalpolicy.org/socecon/glotax/biblio/uscong.htm>.

55 UNDP, op. cit., S. 116. Seit den 90er-Jahren hat die UNO im Übrigen mehrere Forschungsprojekte zu globalen Abgaben aufgegeben. Siehe dazu Martens Jens und Paul James A. The Coffers are not empty: Financing for Sustainable development and the role of the United nations, Juli 1998, $<$ www.globalpolicy.org/sececon/global/paul.htm $>$.

56 $<$ www.nua.ie/ $>$. 
Militärs und der Universitäten von vorwiegend westlichen, gebildeten, jungen und englischsprachigen Männern gemacht, entwickelt und in der Folge auch geprägt. Die Internet-Pioniere hatten - ohne dessen Erfolg abzusehen - ein Instrument für ihre eigenen Bedürfnisse geschaffen.

\section{Drahtlose Versuchung im Süden*}

Als drahtlose Netze werden zahlreiche Datenübertragungstechnologien bezeichnet, die als Trägermedium Radiowellen benutzen. Die Reichweite der Netze ist sehr unterschiedlich: Die WPAN (wireless personal area network) decken einen Umkreis von wenigen Metern ab und dienen zur Verbindung persönlicher Objekte (Telefon, Computer, Tastatur, Maus, drahtlose Kopfhörer...); die WLAN (wireless local area network) haben eine Reichweite von mehreren Dutzend Metern (Lager, Gebäude...); die WMAN (wireless metropolitan area network) erstrecken sich über mehrere Kilometer und bieten Zugangsdienstleistungen auf der Ebene einer Stadt an; die WWAN schliesslich (wireless wide area network) verfügen über eine noch grössere Reichweite. ${ }^{\mathrm{a}}$

Die Netze sind besser unter dem Sammelbegriff Wi-Fi (zusammengezogen aus "wireless fidelity") bekannt. Damit wird der gemeinsame Standard bezeichnet, auf den sich die HardwareProduzenten geeinigt haben. Seit Ende der 90er-Jahre verzeichnen diese Netze bei Unternehmen, Unabhängigen und bei Gemeinschaften grossen Erfolg. Sie wurden zunächst für die Nutzung im privaten Rahmen konzipiert. Heute halten sie im urbanen und ländlichen Umfeld Einzug. Ein anschauliches Beispiel ist das Dorf Nora in Schweden, das über eine Funkverbindung zur nächsten, 8 km entfernten, Stadt ans Internet angeschlossen ist ${ }^{\mathrm{b}}$.

Die grösste Stärke von Wi-Fi liegt in den niedrigen Kosten: Funkausrüstungen sind Massenprodukte, und die Industrie hat sich auf gemeinsame technische Standards verständigt. Auch die problemlose Paarung dieser Systeme mit PCs und die Möglichkeit, flexiblere Regelungen zu vereinbaren als für andere Technologien und Funkdienstleistungen, zählen zu den Vorteilen von Wi-Fi.

Heute ist es möglich, zwei 10 Kilometer voneinander entfernte Dörfer für weniger als 1000 USD miteinander zu verbinden und den Daten- und Gesprächsverkehr ohne wiederkehrende Kosten abzuwickeln. Die Wi-Fi stellen die ökonomischen Modelle der Telekom-Betreiber und ihre Regelungssysteme vor neue Herausforderungen; gleichzeitig erschliesst sie den Ländern des Südens neue Perspektiven. Einige haben das bereits erkannt, z.B. Laos und Vietnam, wo die Ministerien für die Verbindung der Forschungsinstitute und der Verwaltungen auf drahtlose Lösungen setzen. ${ }^{\mathrm{c}}$

* Nach dem Artikel von Alberto Escudero-Pascual „Wi-Fi Opportunity Broadband Wireless Access in Developing Countries", November 2003, verfügbar auf der CD-ROM. Mehrere Artikel und Beispiele für die Nutzung dieser Technologien werden in die CD-Rom integriert.

a $<$ http://www.cru.fr/wl/>.

b <http://www.nora-wireless.org/>.

c Vietnam: <http://csd.ssvl.kth.se/ csd2003-team3>; Laos: http://csd.ssvl.kth.se/ csd2003-team1/>.

Anders bei anderen über „Leitungen“ erbrachten gebührenpflichtigen Dienst- 
leistungen (Radio, Fernsehen, Wasser, Elektrizität), die sich gestützt auf staatliche Politiken ausbreiteten, fiel der Aufschwung des Internets mitten in die Politik der Deregulierung der 90er-Jahre. Diese Jahre zeichneten sich auch durch die Zuspitzung der Ungleichheiten zwischen den entwickelten und den Entwicklungsländern hinsichtlich Produktivität, Technologie, Einkommen, sozialen Einrichtungen und Lebensstandard aus. Die meisten Nutzer bewegen sich nach kommerziellen Regeln im Internet. Folglich ist ein Bruch in der Geschichte der Telekommunikationsdienste festzustellen: Die Ausbreitung des Telegrafen oder des Telefons beruhte von Anfang an auf den Kriterien öffentlicher Dienstleistung und universalem Zugang ${ }^{57}$.

\section{Zwei Schritte nach vorne, einer zurück}

Die Hoffnung auf die Verringerung der digitalen Kluft beruht weit gehend auf der Hypothese, dass dank den Informationstechnologien bestimmte Entwicklungsschritte übersprungen werden können. Häufig werden aber Mittel und Zweck verwechselt. Übersprungen wird zuerst die Technologiephase als conditio sine qua non für den Aufbau der Infrastrukturen, welche Zugang zu den IKT erlauben: Wenn das Telefonnetz mangelhaft ist, kann man direkt zur drahtlosen Technologie übergehen. Solche Prognosen haben sich für die MobiltelefonieInfrastrukturen tatsächlich bewahrheitet. 2002 haben die Mobiltelefone mit über einer Milliarde Abonnenten weltweit die Zahl der Festanschlüsse übertroffen. Damit hat sich der Zugang zur Telekommunikation insbesondere in den Ländern des Südens erheblich ausgeweitet ${ }^{58}$. Beim Internetzugang verhält es sich jedoch anders: Die Voraussetzungen für Erfolge sind komplexer ${ }^{59}$, und die Länder ziehen oft die Strategie der kleinen Schritte vor, um die Entwicklungen unter Kontrolle zu behalten ${ }^{60}$. Trotz der steigenden Nutzerzahl vertieft sich die Kluft weiter. Das Internet bleibt stark zentralisiert.

Es gibt eine Korrelation zwischen dem Volkseinkommen und der Anschlussdichte eines Landes. Das reichste Fünftel der Weltbevölkerung besitzt $86 \%$ des weltweiten Einkommens und stellt $93 \%$ der Internetnutzer. Am entgegengesetzten Ende der Skala verdient das ärmste Fünftel 1\% des globalen Einkommens und macht $0,2 \%$ der Internetnutzer aus ${ }^{61}$. Die Anzahl der afrikanischen Internetnutzer ist zwar zwischen 1997 und 2002 um das Sechsfache angestiegen, aber 1997 wie 2002 entfallen nur 1\% aller Internetnutzer weltweit auf den schwarzen Kontinent, bei grosso modo gleichem Wachstum auf den übrigen Kontinenten ${ }^{62}$. Der südlich der Sahara liegende Teil Afrikas ist im Übrigen deutlich unterver-

57 Für die Geschichte und die Diskussion zum Konzept der „Universaldienstleistung“ wird verwiesen auf Cees J. Hamelink, op. cit., S. 84-87.

58 ITU, Rapport sur le développement des télécommunications dans le monde 2002, Genf, S. 8.

59 Unter anderem: verlässliches Elektrizitätsnetz, Telekommunikationsnetz, Kauf, Wartung und Benutzung von Computern, Modems usw. Einige dieser Sachzwänge lassen sich unter bestimmten Voraussetzungen mit den drahtlosen Technologien, die zwar noch kaum verwendet werden, überwinden (siehe dazu Rahmentext S. 16).

60 Institute for Prospective Technological Studies, op. cit., 2001.

61 UN Economic and Social Council, Development and international cooperation in the twenty-first century: the role of information technology in the context of a knowledge-based global economy, New York, 18. Mai 2000, S. 17.

62 Die Schätzung der Anzahl Internetnutzer ist keine exakte Wissenschaft! Die Angaben variieren deshalb je nach Quelle. Für globale Zahlen wird verwiesen auf <www.nua.com/>. Für präzisere Schätzungen betreffend Afrika siehe vorzugsweise <http://www3.wn.apc.org/africa/>. 
treten. Von den 6 Millionen afrikanischen Internetnutzern leben im Januar 2002 vier Millionen im Maghreb oder in Südafrika (und damit nur zwei Millionen Internetnutzer in Afrika südlich der Sahara).

Allerdings ist der Bruch eher qualitativ als quantitativ. Sobald eine Ursache für technologische Ungleichheiten verschwindet, taucht eine andere auf. $\mathrm{Zu}$ den wichtigsten Herausforderungen zählt der Zugang zu Breitbandanschlüssen. Dabei handelt es sich um eine Voraussetzung für die Nutzung von Anwendungen mit hoher wirtschaftlicher oder sozialer Wertschöpfung, insbesondere in den Bereichen Fernmedizin, Fernunterricht, elektronischer Handel und Telearbeit (d.h. also nicht für E-Mails). Deshalb werden die Eliten des Planeten höchstwahrscheinlich bereits in höhere Sphären des Cyberspace vorgestossen sein, wenn die Massen endlich per Telefon Zugang zum Internet erhalten ${ }^{63}$. Das ist bereits der Fall: Die 400000 Einwohner Luxemburgs verfügen über eine höhere internationale Internet-Bandbreite als die 760 Millionen Afrikaner ${ }^{64}$.

Dabei handelt es sich nur um die Spitze des Eisbergs. In Wirklichkeit ist das Internet in technischer Hinsicht sternförmig um einen Mittelpunkt - die Vereinigten Staaten - aufgebaut, die in den internationalen Zusammenschaltungen eine Schlüsselrolle spielen, weil ihre Bandbreite deutlich über jener der übrigen Welt liegt ${ }^{65} .98 \%$ der überregionalen Vermittlungen werden über die Vereinigten Staaten abgewickelt: Die Verbindungen der Internet-Surfer der südlichen (und europäischen) Länder laufen über die Vereinigten Staaten ${ }^{66}$. Das trägt den ärmsten Ländern, welche bei den amerikanischen Betreibern für die BreitbandVerbindungen zahlen müssen, erhebliche finanzielle Nachteile ein. Ausserdem steht diese Situation in krassem Gegensatz zu den Vereinbarungen über Kosten und Nutzen, welche im Zusammenhang mit dem Telefon durch die Aufteilungsgebühr eingeführt wurden.

Die Produktion von Technologien und Inhalten ist noch elitärer. Nach wie vor konzentriert sich der Kern des technologischen Know-hows auf einige Grossunternehmen und eine geringe Zahl innovativer Akteure ${ }^{67}$. Bei der Produktion des Materials ist festzustellen, dass die räumlich stark konzentrierten Grossunternehmen und innovationsfreudigen Start-ups sich zusammen mit den Dienstleistungsanbietern in einigen wenigen technologischen Knotenpunkten - in der Regel am Rande der Metropolen - ansiedeln und über Telekommunikation und Flugverkehr miteinander verbunden sind. Insofern findet keine undifferenzierte räumliche Verbreitung, sondern eine äusserst selektive Konzentration des Wohlstands der „New Economy“ statt $^{68}$.

Den Inhalt betreffend ist zu beobachten, dass die Internet-Domains sich in der

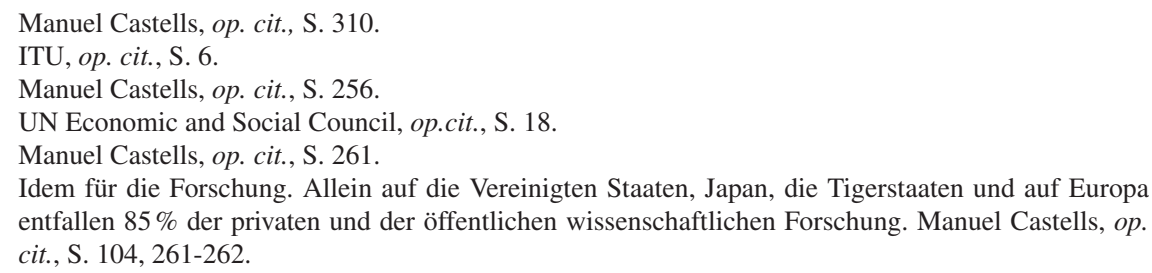


Hand einiger weniger Länder (im wesentlichen Nordamerika und Europa) befinden. Mit 50\% der globalen Domains nehmen die Vereinigten Staaten eine deutliche Vormachtstellung ein. Die Konzentration ist viel stärker als bei den Verbrauchern - ein Zeichen dafür, dass sich die Asymmetrie zwischen der Produktion und dem Verbrauch verschärft: Die Industrieländer produzieren für die restliche Welt (mit Ausname Japans, das deutlich mehr konsumiert als produziert), und die Vereinigten Staaten produzieren für alle anderen ${ }^{69}$.

Kurz: Die Internetnutzung nimmt zwar rasch zu, aber vor dem Hintergrund einer räumlichen Struktur, welche die Geografie nach Wohlstand, Technologie und Macht fragmentiert ${ }^{70}$.

In der ganzen Welt werden die Geschäftsviertel mit modernsten Technologien ausgestattet. Diese Infrastrukturen umgehen das normale Telefonnetz und verbinden die grossen Geschäftszentren, welche die überwältigende Mehrheit der Internet-Verkehrsdaten produzieren und konsumieren, direkt miteinander. Dabei handelt es sich nicht um ein Privileg der Länder des Nordens : Derartige Zentren schiessen gerade in Asien und in Südamerika wie Pilze aus dem Boden (Super Multimedia-Korridor in Malaysia, Hsinchu Science Park in Taiwan, Nova Faria Lima in Sao Paolo, Pudong in Schanghai, San Andres in Lima usw. ${ }^{71}$ ). Das führt in den Städten und Ländern zu tief greifenden Spaltungen und zu dualen Strukturen in wirtschaftlicher, sozialer und kultureller Hinsicht, nach dem Modell der „Festungsviertel“ in Südafrika und Brasilien. Indien besitzt eines der dynamischsten High-Tech-Zentren der Welt, Bangalore (Beiname Boomgalore), doch gleichzeitig beträgt die Analphabetenrate bei Erwachsenen $44 \%$.

Die Ernsthaftigkeit der Argumente für die Beseitigung oder zumindest Verringerung der Ungleichheiten im Zugang zur digitalen Technologie steht ausser Frage. Obwohl die Anschlussdichte der südlichen Länder absolut gesehen zunimmt, ist nicht damit zu rechnen, dass die technologische Kluft bald verschwinden wird. Beim Tempo der technologischen Entwicklung in den Ländern des Nordens - die sich im Übrigen in diesem Bereich seit über dreissig Jahren voluntaristische, staatliche Politiken leisten ${ }^{72}$ - wäre es illusorisch, auf ein Aufholen zu setzen. Warum sollten die gut angeschlossenen Länder auf die anderen warten?

\section{Betrügereien und Illusionen}

Der von der internationalen Entwicklungszusammenarbeit kolportierte Diskurs über das „Aufschliessen“ überzeugt nicht: Damit wird nicht der universale Zugang zu den IKT gefördert, sondern die bereits vorherrschende kommerzielle Expansionsdynamik verstärkt.

Nachdem die Staatsbetriebe in zahlreichen Ländern des Südens ausser Stande

69 Manuel Castells, op. cit., S. 267.

70 Manuel Castells, op. cit., S. 260.

71 Das Magazin Wired identifiziert weltweit rund vierzig „mini-Silicon Valleys“, davon zwei in Afrika (Tunesien und Südafrika), <http://www.wired.com/wired/archive/8.07/silicon.html?pg=1>. Siehe auch Manuel Castells, op. cit., S. 292-93.

72 Hervé Rannou et al. „Les stratégies numériques publiques“ in Nord et Sud numériques, op. cit., S. 301-316. 
waren, hochwertige Telekommunikationsdienste anzubieten, erscheint die Marktöffnung für den Wettbewerb natürlich als unbestrittener Fortschritt. Diese Strategie ist aber aus mehreren Gründen problematisch.

Zunächst sollen die nationalen Regelungen und die staatliche Kontrolle die Entstehung von privaten Monopolen verhindern und die Kontinuität der Verpflichtungen der öffentlichen Dienstleistungen sicherstellen - was jedoch bei weitem nicht der Realität entspricht. Bereits nach einigen Jahren zeichnete sich ab, dass die versprochene Entwicklung der lokalen Märkte nicht stattfand. Konkurse und Verschuldung der Grosskonzerne haben Tausende von Dollars vernichtet, die Preise für die kleinen Nutzer in die Höhe getrieben und Unternehmen im Süden ausgelöscht. Der Fall Senegals, den Amadou Top beschreibt, ist bezeichnend: Die grenzenlose Begeisterung für die IKT, von denen man sich einen Beitrag zur Lösung der Probleme der Entwicklungsländer versprach, ist heute der Ernüchterung gewichen - Ernüchterung angesichts der Raubtierpolitik, welche die grossen internationalen Konzerne im Telekommunikationssektor betreiben, und welche die schlimmsten, teilweise tot geglaubten Formen der Domination aus der Ära der Industriegesellschaft wieder aufleben lassen. Die ultra-aggressiven Methoden der Marktdurchdringung durch die Übernahme der Mehrheitskontrolle zwingt überall zu einer Neuausrichtung der Entwicklungspolitik im Telekommunikationssektor - unter der Fuchtel der neuen Kolonialherren, deren Mission zunächst darin besteht, den Krieg unter den Betreibern des Nordens auf den fragilen Märkten des Südens auszutragen. So investierte France Télécom 70 Milliarden CFA, um Mehrheitsaktionärin von Sonatel zu werden, und verdiente in knapp 4 Jahren mehr als das Doppelte des ursprünglichen Einsatzes, wenn Dividenden, bezahlte Dienstleistungen, Ausrüstungslieferungen usw. auf dem schwach entwickelten senegalesischen Telekommunikationsmarkt hinzugezählt werden $^{73}$.

Die Infrastrukturen der armen Länder mit ausländischen Direktinvestitionen (FDI) und öffentlich-privaten Partnerschaften (PPP) aufbauen und entwickeln $\mathrm{zu}$ wollen, zeugt eher von Wunschdenken, als von ökonomischem Realismus. Das spektakuläre Wachstum der FDI seit den 80er-Jahren geht nicht auf langfristige Investitionen in die ärmsten Länder, sondern auf die Struktur der globalen Oligopole (Fusionen - Übernahmen zwischen Firmen der Industrieländer) und auf Trends zur Rationalisierung des Produktionsapparats der multinationalen Konzerne (höhere Spezialisierung der Produktionseinheiten) zurück. Achtzig Prozent der FDI zirkulieren zwischen Nordamerika und Westeuropa. Auf den afrikanischen Kontinent entfallen knapp 2\% $\%^{74}$. Wie Zentralasien bleibt Afrika von den Finanzflüssen weitgehend unberührt und kann nicht erwarten, den Mangel an Infrastrukturen auf diesem Weg zu beheben.

Die internationale Entwicklungszusammenarbeit im IKT-Bereich verzeichnete in der zweiten Hälfte der 90er-Jahre, als der Nasdaq exponentiell anstieg, einen starken Aufschwung ${ }^{75}$. Die Visionen und Projekte, die heute verwirklicht wer-

Amadou Top, Halte à la prédation, OSIRIS, Montag, 4. November 2002, <www.osiris.sn>

74 Atlas du Monde Diplomatique 2003, Paris, S. 26-27 und Weltbank, The little data book 2002, Washington. 
den sollen - insbesondere die PPP und der WSIS - stammen aus jenen fetten Jahren. Seither (2000-2002) haben sich nach den Börsenkrachs und spektakulären Firmenkonkursen Millionen von Milliarden Dollar durch Spekulationen und irrationale, ja gar betrügerische Investitionen in Luft aufgelöst ${ }^{76}$. Die internationale Zusammenarbeit liess sich davon überrumpeln, ohne ihre Philosophie entsprechend anzupassen. Alternativlösungen für die leeren Versprechen der „New Economy" lassen auf sich warten.

Ebensowenig haben sich die Regeln des Technologietransfers geändert. Unternehmen, die Patente besitzen, befürchten eine Stärkung der Konkurrenz. Sie propagieren die Ausweitung der geistigen Eigentumsrechte, schwächen das Gemeingut und erhöhen die Anzahl der patentgeschützten Innovationen sowie deren Schutzdauer ${ }^{77}$.

Seit den 50er-Jahren hatte sich die Theorie des wirtschaftlichen Catch up ohnehin schon stark abgenutzt. Gilbert Rist äussert sich wie folgt zu der Gewohnheit der internationalen Organisationen, ,zu tun als ob“, um bestimmte Illusionen aufrecht zu erhalten: als ob „Entwicklung“ ̈übertragbar sei, als ob die internationale Schuld zurückgezahlt werden könne, als ob es für die armen Länder möglich sei, zu den reichen aufzuschliessen, als ob grenzenloses Wachstum nachhaltig sein könne, als ob sich virtuell gegen real behaupten könne ${ }^{78}$ - man ergänze: als ob die digitale Kluft überbrückt werden könnte. Selbst wenn man nur einen Moment lang an den technologischen Aufholprozess glauben würde, wie sähe es mit dem wirtschaftlichen Aufholen aus? Wird das Breitband dem Handwerker im Süden erlauben, eine internationale Klientel zu gewinnen, zu beliefern und von ihr bezahlt zu werden?

Bereits bei den „Entwicklern“ der früheren Generation war eine gewisse Achtlosigkeit gegenüber der Vergangenheit und eine Gleichgültigkeit gegenüber früheren Misserfolgen zu beobachten. Die Diagnose Gedächtnisschwund ist im Zusammenhang mit den IKT besonders dramatisch, weil neue, aus den vom technikgläubigen Positivismus stark geprägten Technik- und Industriekreisen stammende Akteure zu denjenigen hinzukommen, die ,,von der Entwicklung der anderen leben“. Umso schneller löscht die von der Informationsgesellschaft ver-

75 Zwischen 1998 und 1999 betrafen sechs der zwölf grössten Unternehmenszusammenschlüsse, bei denen es global um 465,3 Milliarden Dollar ging, Internet-Firmen. 1999 hatte Microsoft 471 Milliarden Dollar kapitalisiert, Intel 285, Lucent 211, Yahoo 188, AOL 164, Oracle 85; im März desselben Jahres erreichte das Kapital von Cisco die Schwindel erregende Summe von 555 Milliarden Dollar fast die Hälfte des jährlichen BIP Frankreichs. Die Magnaten der „New Economy“ rühmten sich, das Tempo der globalen Börsenbewegungen um das Vierfache beschleunigt zu haben. Antonio Pasquali, ehemaliger Vizegeneraldirektor der UNESCO, Sektor Kommunikation <http:// globenet.org/csdptt/ doc_csdptt.php3?id_article=153>.

76 Im Juni 2002 hatten sich nach optimistischen Berechnungen bereits tausend Milliarden Dollar in Nasdaq-Spekulationen verflüchtigt; Pessimisten runden diesen astronomischen Betrag auf viertausend Milliarden Dollar auf. Antonio Pasquali, op. cit.

77 Die Patentanträge steigen exponentiell an. 1999 meldeten die Vereinigten Staaten 169'000 Patente an, im Jahr 1985 waren es erst 77'000 gewesen. Bestimmte Patente betreffen minimale Innovationen, z. B. Patentschutz für die Methode „Einkaufen per Mausklick“ im elektronischen Handel. UNDP, op. cit., S. 103.

78 Rist, op. cit., S. 376. 
heissene Revolution die Erinnerung an die Nord-Süd-Zusammenarbeit der letzten Jahrzehnte.

Ein Fall von Naivität oder Amnesie - oder wird etwas anderes im Schilde geführt?

\section{Inhalte, Wissen und Konsum}

Jede Politik beruht auf der Annahme, dass ein Internet-Anschluss zwangsläufig einen unverzichtbaren, von allen angestrebten Vorteil bedeutet. Internet erzeugt Wohlstand, soziale Bindung und Wissen. Deshalb hoffen alle, früher oder später Zugang zu erhalten. Was aber wird wirklich gewünscht? Ein Arbeitsinstrument, Fernsehen, ein Werbemedium? Zugang zur „Wissens-“ , „Konsum-“ oder „Unterwerfungsgesellschaft"?

\section{Soziale Kontrolle und Marktdisziplin}

Es wäre naiv anzunehmen, dass die Staaten und Unternehmen den universellen Zugang aus reiner Nächstenliebe fördern. Eine Motivation ist auch, die Mehrheit in eine Marktdisziplin einzubinden.

Auf politischer Ebene stellt die von den Beratern des Weissen Hauses entwickelte Soft Power-Doktrin das Internet in die Perspektive der Aussenpolitik: Die amerikanische Demokratie und die freien Märkte führen mit den Informationstechnologien als Multiplikator der Diplomatie eine Charmeoffensive durch. Neue Hoffnungsträger sind dabei die Informationen, die durch Marketing, Fernsehen und Medien, Propaganda, elektronischen Handel sowie strategische Nachrichten gesammelt werden. Das Internet wird zum Erweiterungsvektor für eine friedliche Gemeinschaft von Demokratien und zum besten Garanten einer sicheren, freien und reichen Welt ${ }^{79}$. Soft Power bedeutet damit, andere dazu zu bringen, dass sie das wollen, was man von ihnen möchte: die Fähigkeit, anderen Normen und Institutionen ,schmackhaft zu machen“, die das erwünschte Verhalten herbeiführen; die Fähigkeit, Ziele durch Überzeugung statt durch Zwang $\mathrm{zu}$ erreichen und weniger ökonomische und wirtschaftliche Ressourcen dafür einsetzen zu müssen ${ }^{80}$.

Auf wirtschaftlicher Ebene steht das Konzept „Kapitalismus ohne Reibungsverluste" hoch im Kurs. Im elektronischen Handel werden die Vermittler ausgeschaltet. Verbraucher und Produzent treten zum gegenseitigen Vorteil direkt miteinander in Kontakt. Der Produzent informiert den Käufer direkt über die Dienstleistungen. Im Gegenzug informiert der Käufer den Produzenten über Vorlieben und Gewohnheiten ${ }^{81}$. Ein flüssiger Austausch wird durch die reine und vollkommene Information hergestellt. Mit der Beteiligung aller Verbraucher und aller Produzenten entsteht ein kopfloser, grenzenloser (borderless), aber

79 Armand Mattelard, op. cit., S. 93.

80 Armand Mattelard, op. cit., S. 93 - Bezüglich der Soft Power-Doktrin wird auch auf die Arbeiten von Joseph S. Nye verwiesen, insbesondere Joseph S. Nye, ,Power and Interdependence in the Information Age“, Foreign Affairs, v. 77 Nr. 5 (September/Oktober 1998), New York.

81 Armand Mattelard, op. cit., S. 98. 
auch führerloser (leaderless) Markt. In der sozialen Schwerelosigkeit wird das „Individuum Konsument“ von allen Machtverhältnissen (und von jeglicher Verantwortung) völlig befreit.

Die politischen und wirtschaftlichen Visionen zeitigen technische Konsequenzen. Es kommt zur Konvergenz zwischen Staat und Unternehmen, die aus dem Internet einen mit neuen Spähinstrumenten überwachten Raum machen. Kommerziell wird zunächst identifiziert, wer mit wem worüber kommuniziert. Durch die Installation neuer Software zur Kundenidentifikation wird aus der Information Geld gemacht. Die Staaten führen dabei unter dem Deckmantel sicherheitspolitischer Imperative elektronische Überwachungssysteme ein - voller Enthusiasmus und in der Hoffnung, einen Teil der abbröckelnden Macht zurückzuerobern $^{82}$ - und setzen dabei die Meinungsfreiheit und den Schutz des Privatlebens ihrer Bürger aufs Spiel.

Alle Visionen und technischen Erneuerungen werden abseits des Bereiches der Nord-Süd-Zusammenarbeit entwickelt. Die Akteure der internationalen Zusammenarbeit, die den universellen Zugang propagieren, sind natürlich von globalen Projekten zur Kontrolle ihrer Mitmenschen meilenweit entfernt. Es geht nicht um ein Komplott, sondern um den Glauben an die universalen Tugenden des Konsumgesellschaftsmodells, das mit bestem Gewissen überall in die Welt getragen wird. Diese Vision wurde im Rahmen des Weltbankforums über die Bekämpfung der digitalen Kluft von General Colin L. Powell höchstpersönlich vorgetragen: Wenn die digitale Apartheid nicht abgeschafft wird, werden die Ausgegrenzten der digitalen Ära noch ärmer, noch fortschrittskeptischer und unfähiger, die qualifizierten Arbeiter oder Konsumenten zu werden, die wir brauchen, um das Wachstum der Internet-Wirtschaft zu unterstützen. Die Fortschritte beim Zugang zu digitalen Technologien für benachteiligte Jugendliche in den Vereinigten Staaten zeigen, was machbar ist. Was die Vereinigten Staaten geleistet haben, muss jetzt überall auf der Welt Schule machen. Der Privatsektor besitzt die Motivation, die Ressourcen und den Erfindungsreichtum, um die Welt hin zur Verwirklichung dieses wesentlichen Ziels zu führen ${ }^{83}$.

Das Internet und der elektronische Handel erneuern das Vermögen, beim Menschen unbegrenzte Bedürfnisse wachzuhalten und ständig neue zu wecken, um das eigene Überleben zu garantieren. Der Glaube an die Tugenden der „unsichtbaren Hand“ wird gefestigt. Wie Christian Comeliau vermutet, ist nicht erwiesen, dass der gesellschaftliche Nutzen im Vordergrund steht: [Das System der kapitalistischen Moderne] setzt dazu alle denkbaren Hebel in Bewegung von der Deckung der Grundbedürfnisse und der Hilfe an die Ärmsten über das Fördern des Strebens nach Komfort, Macht oder sozialem Prestige bis zur Organisation der rein konsumorientierten Nachahmung ${ }^{84}$. E-Vakuum

82 Manuel Castells, op. cit., S. 211.

83 "Is the Digital Divide a problem or an opportunity?", Businessweek, 18. Dezember 2000 - Sonderausgabe über die vom World Ressource Institute der Weltbank organisierte Konferenz.

84 Christian Comeliau, Les impasses de la modernité, critique de la marchandisation du monde, Seuil, Paris, 2000, S. 87. 
Das Internet floriert, ohne dass die Frage der Alphabetisierung und Bildung ernsthaft gestellt wird. Das Netz ist im Begriff, zum grössten globalen Werbeträger zu werden. So ist es denkbar, dass uns das Internet die erwarteten Vorteile vorenthält und im Gegenteil Ressourcen ,,verschluckt“”.

An einer von der Weltbank organisierten Konferenz erklärte ein Professor der Michigan-Universität seiner Zuhörerschaft - im wesentlichen Unternehmensleiter - ohne Anflug von Zynismus: Sagen Sie sich beim Anblick der Armen nicht mehr, es gebe keine Hoffnung: Den Armen zu verkaufen, bringt mehr Gewinne, als Ihnen oder mir zu verkaufen. Hier liegt die Zukunft. Die Chancen sind überall. Die digitale Kluft entsteht nicht wegen mangelnder Chancen, sondern wegen mangelnder Phantasie ${ }^{85}$. Man muss also nicht mehr gebildet und gesund sein, um am globalen Markt teilzuhaben - es reicht aus, sich von den vielen Überzeugungsstrategien verführen zu lassen ${ }^{86}$.

Diese Bestrebungen zeigen sich besonders deutlich im Braindrain. Im Jahr 2000 verabschiedeten die Vereinigten Staaten neue Gesetze, um jährlich 200'000 Fachleute - hauptsächlich im Bereich Informatik und neue Technologien - zu „importieren“. Bestimmte europäische Länder sowie Japan verfolgen die gleiche Strategie. Allein in den Vereinigten Staaten wird die Hälfte aller Visa an indische Informatiker vergeben. Laut Schätzungen des UNDP verliert Indien mit den ausgewanderten Fachleuten jährlich 2 Milliarden USD allein an Ausbildungskosten ${ }^{87}$. Ein Viertel der seit 1980 im Silicon Valley gegründeten Informatikfirmen wird von Chinesen und Indern geführt. Gleichzeitig liegt die InternetDurchdringungsrate in Indien nur bei rund 0,1\% $\%^{88}$.

\section{Die Wissensgesellschaft ist gefordert}

Zu Zeiten der Privatisierung der „Leitungen“ und deren Inhalten machen gegenläufige Kräfte eine schlechte Figur. Serge Halimi vertritt sogar die Auffassung, dass die Journalisten der dominierenden Medien nicht mehr Einfluss auf die Information haben als die Kassiererin im Supermarkt auf die Geschäftsstrategie ihres Arbeitgebers ${ }^{89}$. Die IKT dienen jedoch nicht nur zur Verbreitung einer Pädagogik der Unterwerfung. Die Konnektivität nimmt überall zu. Im Internet werden nicht nur Spiele, Werbung und Pornografie verkauft, sondern auch öffentliche Informationen, Ausbildungsmodule, Dissertationen und Forschungsergebnisse präsentiert.

Die IKT verändern weltweit die Voraussetzungen für den Zugang zur Informa- 
tion. Zahlreichen Berufen, in denen sich das Wissen rasant schnell ändert, bringt Internet klare Vorteile. Der Zugang zu medizinischen, juristischen, wissenschaftlichen Datenbanken usw. ermöglicht Verbesserungen in der Praxis, vorausgesetzt, dass die Daten übernommen werden können. Die Informationsmenge ist nicht spontan für alle „nutzbar“. Der Zugang zu sämtlichen Informationen ersetzt nicht die Vorkenntnisse, die erforderlich sind, um zu beurteilen, welche Information gesucht und wie sie eingesetzt werden soll. Der Fortschritt ist durch die vorher erworbenen Kompetenzen konditioniert, welche Wissen hervorbringen können ${ }^{90}$. Es ist überheblich, zu meinen, Bildung entstehe durch den Zugang zum Netz von selbst ${ }^{11}$.

Wie wertvoll sind leistungsfähige Instrumente, wenn gleichzeitig die Daten immer stärker segmentiert sind? Das Modell der offenen Wissenschaft und der globalen wissenschaftlichen Zusammenarbeit wird womöglich durch das geistige Eigentumsrecht und durch Schutzmassnahmen, die aus der engen Verflechtung zwischen öffentlicher und privater Forschung hervorgehen, unterlaufen. Die Tendenz zur Privatisierung der Aktivitäten und der wissenschaftlichen Entdeckungen hat zahlreiche Institutionen, die in den Industrieländern eine offene Wissenschaft praktizieren, veranlasst, den Zugang zu ihren Arbeiten sowie die Kommunikation unter Wissenschaftlerkollegen aus den entwickelten Ländern wie den Entwicklungsländern einzuschränken ${ }^{92}$. Die IKT verstärken die Abschottung der Wissenschaftler des Südens in immer engeren, vom Markt aufgezwungenen Schranken. Neben den Problemen, die mit der Forschung in ihren Ländern zusammenhängen, stossen die Wissenschaftler des Südens auf den Merkantilismus der westlichen Länder, welcher den Braindrain noch verschärft.

Die Verheissungen der „Wissensgesellschaft“ richten sich nicht nur an Wissenschaftler, sondern über das Konzept der „E-Demokratie“ an die Bürger im Allgemeinen. Erfahrungsgemäss passt sich das Internet an autoritäre Systeme an ${ }^{93}$. Trotzdem ist zu hoffen, dass die IKT die Aufgabe derjenigen erschweren, die diktatorische Neigungen hegen, und dass sie die politische Beteiligung der Bürger überall erleichtern. Diversifizierung der Quellen, Zugang zu öffentlichen Informationen, mögliche Interaktivität zwischen Regierenden und Bürgern und Schaffung von Bürgernetzen: Dies sind nur einige der Potenziale, welche die demokratische Debatte und die Praxis beleben.

Die Nachfrage hängt indessen mit der individuellen gesellschaftlichen Stellung zusammen. Wie Dominique Wolton betont, besteht eine Konsequenz der soziokulturellen Domination gerade darin, nicht mehr zu wünschen, als was man hat; wer etwas anderes will und aktiv wird, befindet sich bereits in der dynamischen Aktion des Infragestellens und der Emanzipation. Die Gefahr besteht darin, dass es zwar Platz für alle gibt, dass aber jeder an seinen Platz gehört ${ }^{94}$.

90 Hansson Sven Ove, „Les incertitudes de la société du savoir“, in La société du savoir, Revue internationale des sciences sociales, Nr. 171, UNESCO/ERES, März 2002, S.4 4.

91 Dominique Wolton, op. cit., S. 89.

92 Forefo-Pineda Clemente, Jaramillo-Salazar Herman, „L'accès des chercheurs des pays en développement à la science et à la technologie internationales", in La société du savoir, op. cit., S. 151.

$93<$ www.rsf.fr/>, Rubrik ,Internet“". 
Das Internet ist in politischer und sozialer Hinsicht nutzlos, wenn die Einzelpersonen es sich nicht zu Eigen machen und damit über die Rolle des Konsumenten hinausgehen ${ }^{95}$.

Cees J. Hamelink folgt der gleichen Argumentation und bietet Denkansätze an, die den IKT Sinn verleihen sollen: Er schlägt vor, Pierre Bourdieus Liste der „Kapitalarten“ um das „Informationskapital“ zu erweitern. Das Konzept umfasst ökonomische Kapazitäten für den Netzzugang, technische Kompetenzen für das Beherrschen der elektronischen Instrumente, intellektuelle Kapazitäten für das Recherchieren, Sortieren und Analysieren der Information sowie die Motivation, aktiv Informationen zu suchen und sie in soziale Praktiken umzusetzen ${ }^{96}$. Dieses Kapital ist in den verschiedenen Gesellschaften sehr ungleich verteilt.

Den Eindruck zu erwecken, der Zugang (über Internet) zum „,universalen Wissen“ werde den sozialen Bruch kitten, zeugt von einem auf „Verbreitung“ basierenden, überholt geglaubten Entwicklungskonzept. Der Nutzen des „Self-Service-Wissensprojekts" ist nicht erwiesen. Um mit Bildung, Kultur und Geld die Klippen der Segmentierung der Inhalte zu umschiffen, sind umfassende und komplexe Anstrengungen vonnöten, die uns unweigerlich zurück zu den ungelösten Fragen der „Entwicklungsära“ führen.

\section{Mehr als Slogans}

Keine zehn Jahre nach dem Start des Weltbankprogramms Information for development herrscht eine auffallende Diskrepanz zwischen dem ungebrochenen Enthusiasmus der institutionellen Kreise und den wenig überzeugenden Lösungen, die sie vorschlagen, um die IKT in den Dienst des guten Lebens für alle zu stellen. Kann der propagierte Kampf gegen die digitale Kluft in diesem Kontext wirklich ernst genommen werden?

„Digitale Kluft“ klingt nach einem weiteren kurzlebigen und zu Mobilisierungszwecken kreierten Slogan, auf den sofort der nächste folgt, sobald klar wird, dass die Versprechen nicht eingehalten wurden. Ein paar Jahrzehnte lang wird der digitale Graben der internationalen Zusammenarbeit, die ihre Missionen neu darauf ausrichtet, einen zweiten Frühling bescheren. Die Instrumentalisierung des Slogans durch die Staaten und die Grosskonzerne, welche die Schäden der „New Economy“ verschleiern und gleichzeitig die rückläufigen Mittel für die internationale Solidarität rechtfertigen, bedeuten aber auch sein sicheres Ende.

Nehmen wir die Hypothese an, nach welcher das Internet in den Mittelpunkt unserer „soziotechnischen“ Gesellschaftsorganisation rückt, sind die Perspektiven düster ${ }^{97}$. Zum Glück aber wird die Geschichte nicht im Voraus geschrieben. Der wahrscheinliche Misserfolg der Programme zum Kampf gegen den digita-

94 In diesem Sinne sind Massenmedien wie Fernsehen, Zeitungen oder Radio demokratischer: Es sind Kommunikationsinstrumente, die allen das Gleiche geben. Der Zugang ist weniger segregiert, obwohl auch Ungleichheiten vorkommen. Dominique Wolton, op. cit., S. 99.

95 Valérie Peugeot, Militantisme et technologies de l'information et de la communication: vers un laboratoire du changement sociétal ?, August 2002, <www.vecam.org>.

96 Cees J. Hamelink, op. cit., S. 91. 
len Graben erweist sich vielleicht als Chance, weil nämlich die Perspektive auf eine höchstmögliche Ausweitung der Marktdisziplin von den Idealen Gleichheit, Demokratie und Bürgertum, denen sie nur als Feigenblatt dient, meilenweit entfernt ist.

Wenn die internationale Zusammenarbeit sich nicht von der binären Logik befreit, auf der das Konzept des digitalen Grabens beruht, erscheint es fragwürdig, eigene - auch nur geringe - Ressourcen in den IKT-Bereich zu investieren. $\mathrm{Zu}$ behaupten, der digitale Bruch liesse sich kitten, ist unwahr. Trotzdem entstehen Nutzungsnischen für diejenigen, die vom Hochgeschwindigkeitsnetz ausgegrenzt sind. Das sozioökonomische Potenzial der IKT ist durchaus vorhanden, und die historischen Merkmale des Internets sind nicht für alle Zeiten in Stein gemeisselt. Glücklicherweise sind die Praktiken der Internet-Nutzung bis heute noch nicht vereinheitlicht worden.

Die Verbreitung, auch wenn sie ungleich ist, macht das Internet zum Medium mit originellen Inhalten, die der Vielfalt der Menschen sowie den geografischen und institutionellen Verhältnissen gerecht wird. Informationen verbreiten und Wissen teilen bedeutet auch, ,zivile Gegenexpertise“ zu entwickeln, welche die Technologie (vor allem mit Gratis-Software), die Methoden (mit der Schaffung und Moderation von Netzen, der Produktion kollektiven Wissens usw.) und die Politik (neue Forderungen, Mobilisierung und Partnerschaften für Projekte, usw.) beeinflusst. Die entsprechenden Anliegen des Staatsbürgers der „Informationsära" verdienen Unterstützung, vorausgesetzt, dass sie die Forderungen der „Agrarära“ und der ,industriellen Ära“ - welche nolens volens für die meisten Bewohner des Planeten nach wie vor Realität sind - nicht aufheben, sondern mit aufgreifen.

97 Der digitale Bruch misst sich nicht an der Anzahl der Personen mit Internet-Anschluss, sondern an den simultanen Auswirkungen der Verbindung und der Nicht-Verbindung. Internet ist nicht nur eine Technologie, sondern ein Instrument und eine organisatorische Struktur zur Verteilung der Informationsmacht, der Schaffung von Wissen und der Fähigkeit zur Vernetzung in allen Bereichen. Manuel Castells, op. cit., S. 326. 